\title{
A Clue to Understand Environmental Influence on Friction and Wear of Diamond-Like Nanocomposite Thin Film
}

\author{
Sukhendu Jana, ${ }^{1}$ Sayan Das, ${ }^{1}$ Utpal Gangopadhyay, ${ }^{1}$ Anup Mondal, ${ }^{2}$ and Prajit Ghosh ${ }^{1}$ \\ ${ }^{1}$ Meghnad Saha Institute of Technology, Techno India Group, Kolkata 700150, India \\ ${ }^{2}$ Department of Chemistry, Bengal Engineering and Science University, Howrah 711103, India \\ Correspondence should be addressed to Prajit Ghosh; dr_p_ghosh@rediffmail.com
}

Received 24 September 2012; Revised 24 January 2013; Accepted 27 January 2013

Academic Editor: Qian Wang

Copyright (c) 2013 Sukhendu Jana et al. This is an open access article distributed under the Creative Commons Attribution License, which permits unrestricted use, distribution, and reproduction in any medium, provided the original work is properly cited.

\begin{abstract}
The wear and friction of diamond-like nanocomposite (DLN) film have been investigated in air with different relative humidity (RH), under deionized (DI) water and saline solution. The structure of the film has been characterized by Fourier transform infrared (FTIR), Raman spectroscopy, and scanning electron microscope (SEM). The result shows two interpenetrating network structure: $\mathrm{a}-\mathrm{C}: \mathrm{H}$ and $\mathrm{a}-\mathrm{Si}: \mathrm{O}$, and they are interpenetrated by $\mathrm{Si}-\mathrm{C}$ bonding. The tribological performance has been measured using ball-ondisc tribometer with tungsten carbide ball as counterbody at $10 \mathrm{~N}$ normal load. Results show that with increasing relative humidity $(\mathrm{RH})$ from $35 \%$ to $80 \%$, the coefficient of friction (COF) increases gradually from 0.005 to 0.074 , whereas with increasing $\mathrm{RH}$ the wear factor decreases from $9.8 \times 10^{-8} \mathrm{~mm}^{3} / \mathrm{Nm}$ and attains a minimum value of $2.7 \times 10^{-8} \mathrm{~mm}^{3} / \mathrm{Nm}$ at $50 \%$ RH. With further increase of RH the wear factor increases again. Moreover, in DI water and especially in saline solution, both the COF and wear factor have been found to be significantly low. A clue has been interpreted to understand environmental dependency, considering the effect of surface dangling bonds, charge transfer, and chemical interactions.
\end{abstract}

\section{Introduction}

Diamond-like nanocomposite (DLN) film comprises of two amorphous interpenetrating network structures: one is "diamond-like" (a-C:H) network, and the other is "glasslike" (a-Si:O) network [1-4]. The presence of a-Si:O network as a reinforcement matrix distinguishes the DLN film from conventional diamond-like carbon (DLC) film $[5,6]$. The material possesses a number of unique bulk and surface properties like hardness with flexibility [7], thermal stability $[7,8]$, corrosion and wear resistance $[8,9]$, biocompatibility $[10,11]$, and so forth. The low residual stress and good adherence to any type of substrates make DLN film a potent material for a variety of tribological applications.

It is believed that the tribological properties of the film are not only inherent properties of the film. They also strongly depend on the surrounding environment and counterbody. Many researchers have reported the dependency of environment on tribological behavior of DLC film $[12,13]$, though limited reports have been published particularly for DLN film. Neerinck et al. have reported that the COF of the DLN film against steel ball at 50\% RH varied from 0.04 to 0.08 and remains less than 0.1 even at $90 \% \mathrm{RH}$. They have also observed that film wear factor was extremely low under water [14]. Scharf et al. have also reported that COF increased from 0.02 to 0.2 when the $\mathrm{RH}$ changed from $1 \%$ to $50 \%$ [15]. However, continuous variation of $\mathrm{RH}$ and presence of ionic solution effect on tribological properties have not been yet reported.

In the present study, the wear factor and COF of DLN film on glass substrate have been studied using standard ball-on-disc tribometer with a tungsten carbide (WC) ball as counterface material under different ambients like air with different $\mathrm{RH}$, under DI water, and saline solution. A clue for environmental dependency on tribological properties of DLN film has been interpreted, considering the films surface dangling bond passivation with environmental species and hence interaction with counterbody.

\section{Experimental Details}

2.1. Synthesis of DLN Film. The plasma-assisted chemical vapour deposition (PACVD) system exploited in the present 


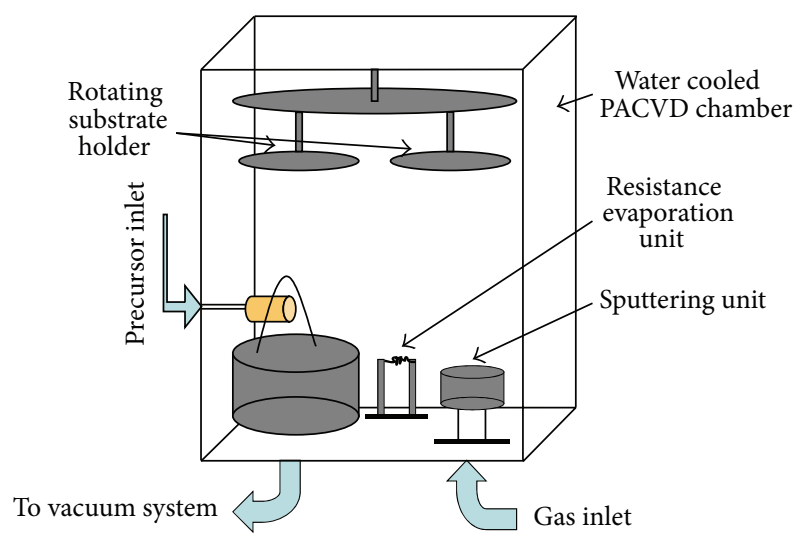

FIGURE 1: Schematic diagram of PACVD system for DLN thin film deposition.

study has been illustrated schematically in Figure 1. The substrates were cleaned by acetone and the alcohol with ultrasonic vibration, followed by drying in a nitrogen jet. Cleaned substrates were loaded into the PACVD chamber followed by evacuation up to a pressure of about $10^{-5}$ mbar. Thereafter, argon gas was introduced into the chamber via a mass flow controller until the chamber pressure reached approximately $10^{-4}$ mbar. The samples were further cleaned in situ by argon plasma for 5 minutes prior to DLN film deposition. During deposition, the filament current was maintained at about $100 \mathrm{~A}$, with a voltage of about $10 \mathrm{~V}$. The thermoionic electrons emitted from the filament were drawn towards the ground at zero potential by applying a voltage of about $-142 \mathrm{~V}$ to the floating filament with respect to ground. The precursor flow was adjusted via a needle valve and by using gravity control. The precursor ejection head was adjusted below the filament, such that on evaporation the precursor molecules come in the path of thermionic electrons. The precursor vaporized due to low pressure and high temperature near the filament in electron atmosphere which enhanced ionization of vapor molecules by collision. The RF substrate bias power supply was concurrently switched on. The precursor ions formed the stable plasma (the plasma current could be adjusted from $0.2 \mathrm{~A}$ to $20 \mathrm{~A}$ ), and the ions accelerated towards the substrates due to the negative DC substrate bias induced by the RF power. A crossed magnetic field was applied using an electromagnet to induce spiraling motion, in order to increase the path length of ions. For the present study, DLN film was deposited on glass substrates in a specially designed PACVD system, using a liquid precursor $(2,3,4-$ triphenyl nonamethyl pentasiloxane) containing $\mathrm{C}, \mathrm{H}, \mathrm{Si}$, and $\mathrm{O}$ as constituents. A typical growth condition has been given in Table 1. During deposition, sputtering and resistance evaporate units were shut down. The film was deposited on glass for $1 \mathrm{hr}$.

2.2. Tribological Measurement. The wear and friction characterization was performed using ball-on-disc tribometer (Figure 2). The samples were fixed on a metal disc which rotated with $200 \mathrm{rpm}$. A WC ball of $4 \mathrm{~mm}$ diameter with $10 \mathrm{~N}$

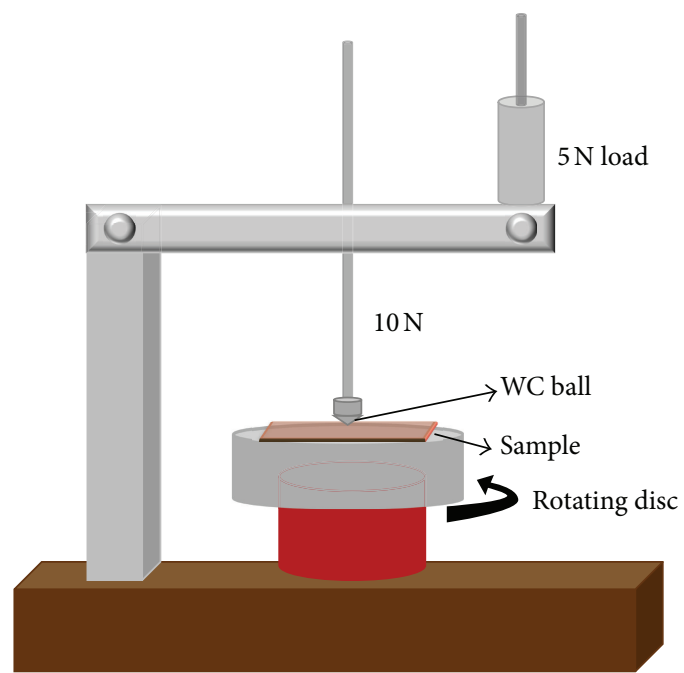

FIGURE 2: Schematic diagram of ball-on-disc tribometer with $10 \mathrm{~N}$ normal load and tungsten carbide ball.

normal load touched the film surface. The distance between WC ball and center of the disc was $4 \mathrm{~mm}$. So the diameter of the wear track was $8 \mathrm{~mm}$, and the linear speed of the ball over the film surface was $0.08 \mathrm{~m} / \mathrm{s}$. At the initial stages of wearing process, the COF was usually high but after a few revolutions, the $\mathrm{COF}$ decreased and reached a low and stable value. Again it increased abruptly at the instant; the film was completely worn off, resulting from the contact of the WC ball tip with the substrate surface. The wear test was performed at room temperature. Each wear test was carried out until the WC ball reached the base substrate, and each observation was measured three times and confirmed.

The wear factor is defined as the amount of volume $\left(\mathrm{mm}^{3}\right)$ wear out per unit normal force $(\mathrm{N})$, per unit sliding distance (m). In ideal case, the cross section of the wear track is a circular curvature due to WC ball as counterbody as has been shown in Figure 3. But in our experiment, the film thickness was very much smaller than the wear track width (Figure 4), and the wear test was performed until completely worn out of the film. So a rectangular cross section of the wear track has been assumed. The total surface area of the wear track is $\pi\left(r_{1}^{2}-r_{2}^{2}\right)$ where $r_{1}$ and $r_{2}$ are the external and internal track radius, respectively.

The total worn-out volume is $\pi\left(r_{1}^{2}-r_{2}^{2}\right) t$ where $t$ is the thickness of the film. The coating wear factor $k$ is expressed as

$$
k=\frac{\pi\left(r_{1}^{2}-r_{2}^{2}\right) t}{F s} .
$$

Here, $F$ is the normal force $(10 \mathrm{~N})$, and $s$ is the total sliding distance over DLN film. The internal and external wear track radius was measured by a high resolution optical microscope (Figure 4). A high temperature thermal adhesive tape was attached before the film deposition on glass substrate to measure the film thickness. The thickness of the film was estimated using a stylus surface profilometer. The resolution of the instrument was $1 \mathrm{~nm}$, and the film thickness is $1093 \mathrm{~nm}$. 
TABLE 1: Deposition condition for DLN film.

\begin{tabular}{lcccccc}
\hline Bias voltage & Plasma current & Precursor flow rate & Ar flow rate & Magnetic field & RPM of substrate & Working pressure \\
\hline $550 \mathrm{~V}$ & $0.8 \mathrm{~A}$ & $0.756 \mathrm{~mL} / 10 \mathrm{mins}$ & $50 \mathrm{~mL} / \mathrm{min}$ & $120 \mathrm{Gauss}$ & 3 & $7.5 E-5 \mathrm{mbar}$ \\
\hline
\end{tabular}

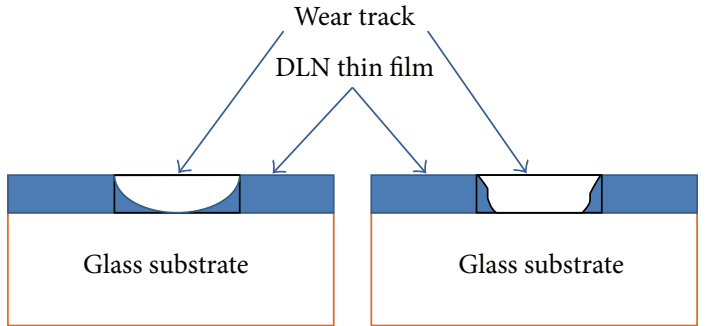

(a)

(b)

FIGURE 3: Schematic diagram of cross-sectional wear track of DLN film for (a) ideal case and (b) assumed.

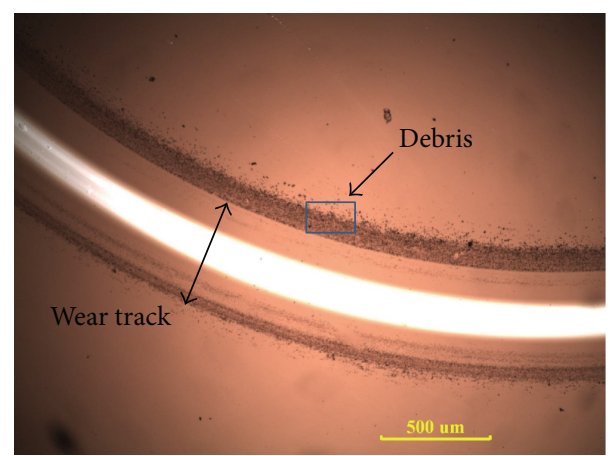

FIGURE 4: Optical microscope image of the wear track of the $1.09 \mu \mathrm{m}$ thick DLN film at $35 \% \mathrm{RH}$ ambient shows the track width $483 \mu \mathrm{m}$.

The total number of revolutions divided by the measured thickness of the film is the wear rate (cycles/micron) of film.

\section{Results and Discussion}

3.1. Film Structure Analysis. In order to study the local bonding of $\mathrm{C}, \mathrm{H}, \mathrm{Si}$, and $\mathrm{O}$ sites of the DLN film, the FTIR spectrum of a representative film was recorded from 400 to $4000 \mathrm{~cm}^{-1}$ range with $4 \mathrm{~cm}^{-1}$ resolution and 100 scan. The corresponding FTIR trace has been shown in Figure 5. The spectra shows a strong $\mathrm{Si}-\mathrm{O}$ absorption peak which had appeared around the wave number of $1100 \mathrm{~cm}^{-1}$ [12]. In the range of $682-885 \mathrm{~cm}^{-1}$, broad absorption band is found which corresponds to $\mathrm{Si}-\mathrm{C}, \mathrm{Si}-\mathrm{CH}_{3}$ fragments $[16,17]$. The mode at $760 \mathrm{~cm}^{-1}$ is due to $\mathrm{Si}-\mathrm{CH}_{3}$ rocking/wagging or $\mathrm{Si}-$ C stretching $[16,17]$. The absorption band at $\sim 857 \mathrm{~cm}^{-1}$ is attributed to $\left(\mathrm{SiH}_{2}\right)_{n}$ bending $[12,13]$.

An absorption band has appeared in the range of 2000$2300 \mathrm{~cm}^{-1}$ which corresponds to $\mathrm{Si}-\mathrm{H}$ and $\mathrm{Si}-\mathrm{H}_{2}$ stretching. The $\mathrm{C}=\mathrm{C}$ stretching vibration has shown its presence with an absorption band from 1372 to $1970 \mathrm{~cm}^{-1}$, but only a broad spectrum from 1370 to $1650 \mathrm{~cm}^{-1}$ is clearly seen in the IR

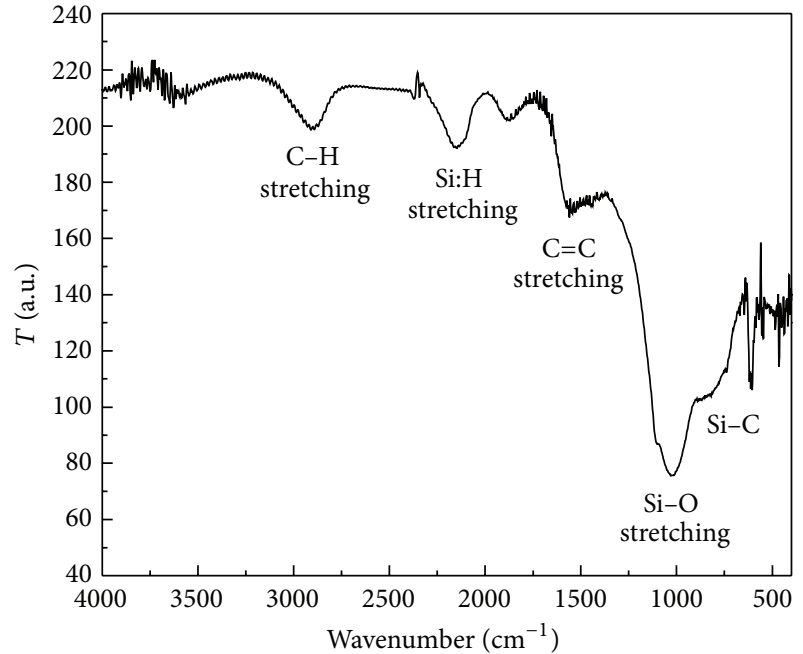

FIGURE 5: FTIR spectrum of the film on glass substrate shows typical diamond-like nanocomposite structure.

spectra, and some $\mathrm{C}=\mathrm{C}$ bands might have been covered up by the moisture noise, which occurs at around $1885 \mathrm{~cm}^{-1}$. A broad spectrum due to $\mathrm{C}-\mathrm{H}$ stretching has occurred at 2750 $3100 \mathrm{~cm}^{-1}$. [16, 17]. The FTIR trace of the film shows two networks: one is diamond-Like $\mathrm{C}: \mathrm{H}$ network and another is Si:O network, and they have been interpenetrated with the $\mathrm{Si}-\mathrm{C}$ bonding depicting the typical nature of DLN film.

In order to understand the structure of DLN film the Raman spectrum was investigated in the wavenumber ranging from 400 to $2500 \mathrm{~cm}^{-1}$. Raman spectrum analysis of DLN film wasdeconvoluted into two Gaussian peaks: the G peak and D peak by curve fitting (Figure 6). The Raman spectrum corresponding to single crystal diamond has a sharp peak located at $1332 \mathrm{~cm}^{-1}$ [18], while that for single crystal graphite has a sharp peak at $1580 \mathrm{~cm}^{-1}[18,19]$. The diamond peak has associated with the fourfold $\mathrm{sp}^{3}$ hybridized $\mathrm{O}_{\mathrm{h}}$ symmetry, while the graphite peak has associated with the threefold $\mathrm{sp}^{2}$ coordination [18-21]. The $\mathrm{G}$ peak, which may occur from $\mathrm{C}=\mathrm{C} \mathrm{sp}^{2}$ stretching vibration of olefinic or conjugated carbon chains, is attributed to the relative motion of $\mathrm{sp}^{2}$ hybridized carb,on and the down shift of the G peak is related to bond angle disorder [22-24]. The D peak is attributed to the disordered breathing motion of sixfold aromatic rings $[18,22]$ Thus, the peak positions in the Raman spectrum of DLN film and the intensity ratio of D/G peak are the most important parameters to understand the bulk properties of the DLN film for tribological purpose. There are two bands around $1355 \mathrm{~cm}^{-1}$ (D peak) and $1524 \mathrm{~cm}^{-1}$ (G peak). The position of $D$ peak at $1355 \mathrm{~cm}^{-1}$ and down shift of the $G$ line indicate the increase in the number/size of $\mathrm{sp}^{2} \mathrm{C}=\mathrm{C}$ bonds with bond angle disorder in the DLN film deposited on glass 


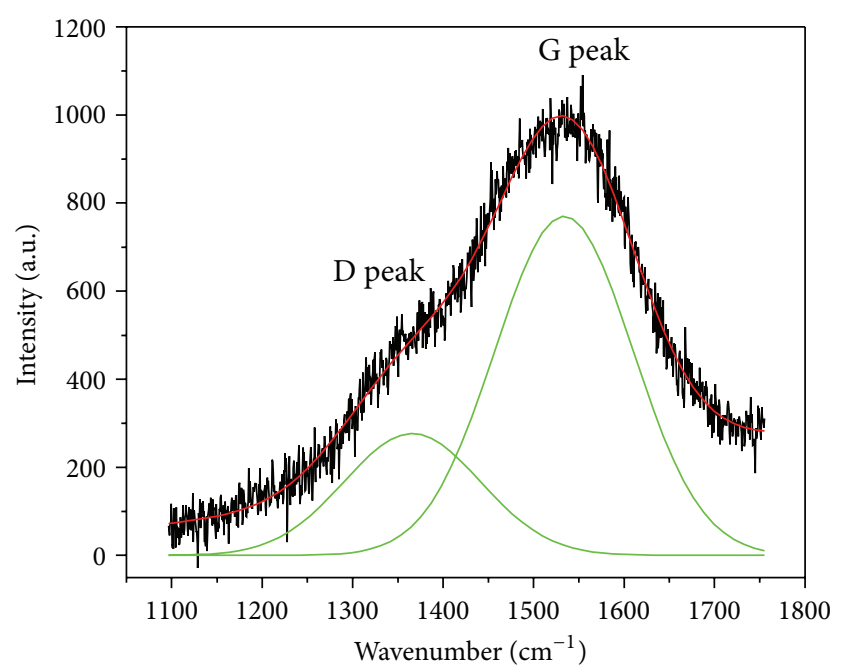

FIGURE 6: Raman spectrum of DLN film on glass substrate deconvoluted at two Gaussian peaks: D peak and G peak.

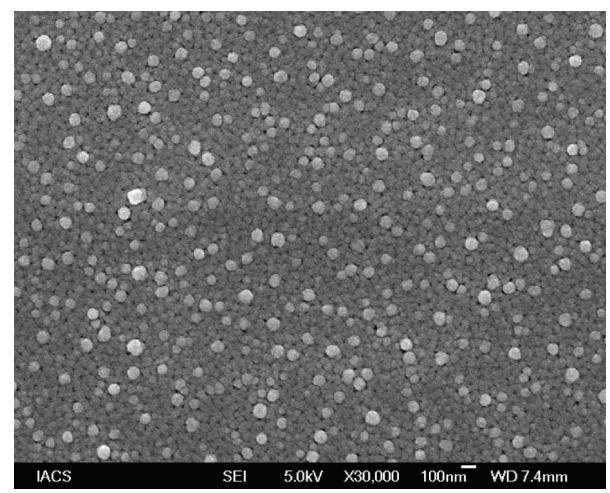

FIGURE 7: FE-SEM image of DLN film surface on glass substrate shows granular like matter distributed randomly.

substrate. Again the intensity ratio $\mathrm{I}_{\mathrm{D}} / \mathrm{I}_{\mathrm{G}}$ varies inversely with the graphite cluster size $[22,23]$. In this respect the ratio, $\mathrm{I}_{\mathrm{D}} / \mathrm{I}_{\mathrm{G}}$, may be indirectly related to $\mathrm{sp}^{3} / \mathrm{sp}^{2}$ intensity, and it is observed to be 0.35 from Gaussian fitting after base line correction.

Figure 7 shows the FE-SEM image of DLN film deposited on glass substrate. Before capturing the image, a very thin layer of gold film was deposited on film surface to nullify the charging effect. The film comprised of amorphous granular like matter, and they were distributed randomly. The average size of these grains was around $75 \mathrm{~nm}$.

3.2. Wear and Friction Characterization. The COF and wear factor of DLN film deposited on glass substrate in different ambient condition like air environment for different relative humidity starting from $35 \% \mathrm{RH}$ to $80 \% \mathrm{RH}$ under water and saline solution were measured. The COF for a particular wear test was not fixed at a particular value. It was varying within a certain range. The average COF values were obtained by averaging the friction coefficients measured in tribometer during the wearing process of the film. Figure 8 shows the

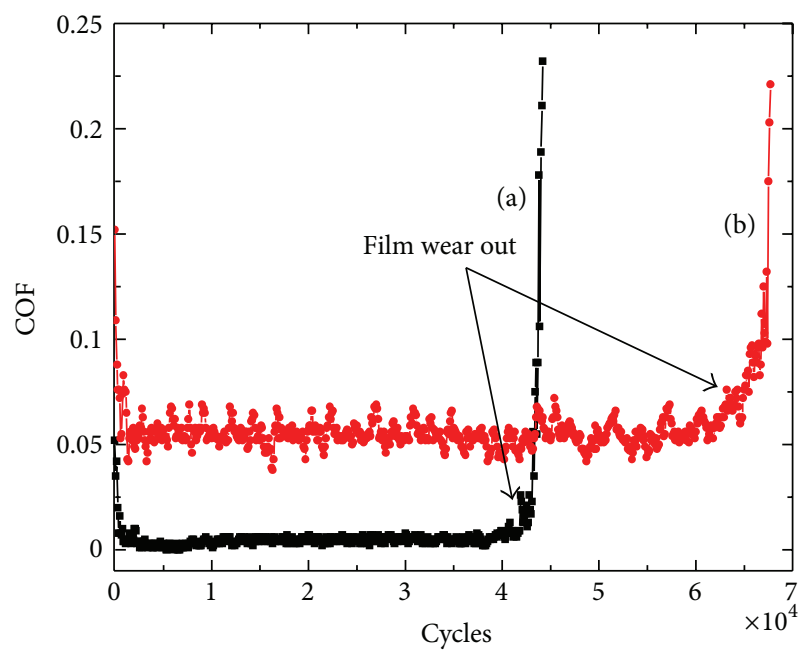

FIGURE 8: Measurement of the coefficient of friction, in a ball-ondisk test with $10 \mathrm{~N}$ load, of DLN film in air with (a) $35 \% \mathrm{RH}$ and (b) $60 \% \mathrm{RH}$.

measurement of COF of DLN film against WC ball in presence of air with $35 \% \mathrm{RH}$ and $60 \% \mathrm{RH}$. The results indicate that, initially the COF was high, after few cycles it reached a stable value, and finally it increased abruptly after the completely wearing out of the film. The DLN film after deposition exhibited surface undulation in the nanoscale (Figure 7). When the TC ball in the tribometer started to slide over the surface of DLN film, the top of the granular structure was gradually flattened due to wearing effect, and the track surface became smoother resulting in the fall of COF from the initial value. The obvious reason was that the crests come in close proximity to the sliding ball surface. Consequently the dangling bonds present on DLN surface and on the crests came in closest proximity of the ball surface. The ball being metallic, electron transfer occurred, and relatively strong interaction and adhesion set in between the DLN film and the ball surface. This strong interaction helped to wear down the crests during sliding of ball.

When the surface became flatter, the sliding ball surface came in close proximity to much larger number of dangling bonds than only on the crest to show the following effects. As the number of interacting dangling bonds increased, the top layer of DLN surface stuck to the ball resulting in high wear factor of DLN film. Furthermore the peeled off fragments came on the wear track to induce abrasive wear which in turn enhanced the wearing of the film $[15,25]$.

The data from Figure 9 suggests that although the COF increased gradually with increase in relative humidity $(\mathrm{RH})$, the value of COF was very low $(<0.08)$ even in high humid condition ( $\mathrm{RH} 80 \%)$. Another interesting observation was that the COF under DI water was less than that in high humid air (RH 80\%) and was further reduced to 0.021 in saline solution which was close to that in $50 \% \mathrm{RH}$ condition. The data collected from the wearing process have been listed in Table 2, and the corresponding results on the wear factor for different ambient conditions have been shown in Figure 10. 


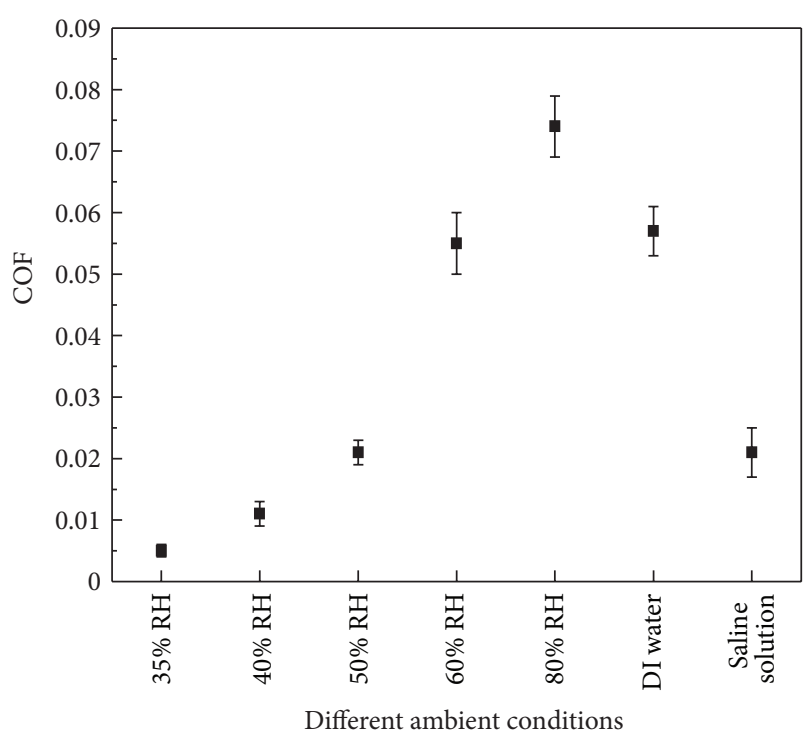

FIGURE 9: COF of DLN film on glass substrate in different ambient that is, $35 \% \mathrm{RH}$ air; $40 \% \mathrm{RH}$ air; $50 \% \mathrm{RH}$ air; $60 \% \mathrm{RH}$ air; $80 \% \mathrm{RH}$ air; DI Water; $0.9 \% \mathrm{NaCl}$ aqueous soln.

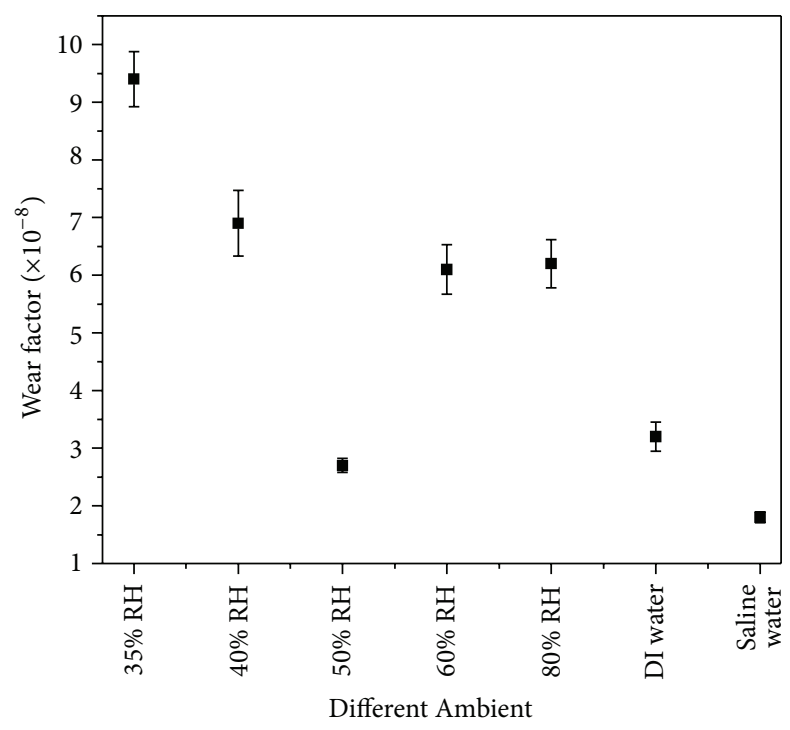

FIGURE 10: Wear factor of DLN film on glass substrate in different ambient that is, $35 \% \mathrm{RH}$ air; $40 \% \mathrm{RH}$ air; $50 \% \mathrm{RH}$ air; $60 \% \mathrm{RH}$ air; $80 \% \mathrm{RH}$ air; DI Water; $0.9 \% \mathrm{NaCl}$ aqueous soln.

In this context it might be note that the wear factor of DLN film decreased with increase in humidity of air from $\mathrm{RH}$ $35 \%$ to RH $40 \%$ and attained a sharply low value at RH 50\%. Further observation revealed that the wear factor rose again at humidity higher than $\mathrm{RH} 50 \%$, attained relatively higher values again at $\mathrm{RH} 60 \%$, and remained more or less unaffected by humidity till RH $80 \%$. But under DI water and under $0.9 \%$ $\mathrm{NaCl}$ solution the wear factor maintained an extremely low value (Table 2 and Figure 10). As discussed previously, our observations can be summarized from Figures 9 and 10 and Table 2 as (a) both the COF and wear factor were low under $0.9 \%$ $\mathrm{NaCl}$ solution;

(b) the COF was high but wear factor was relatively low under DI water;

(c) the COF was very low and wear factor rather high in low humidity air with $\mathrm{RH}<40 \%$;

(d) the COF and wear factor both were high at high humid condition at RH $80 \%$.

Above-mentioned frictional and wear behavior could be explained by the consideration of electrical and chemical effects during the process of measurements.

Further Figure 10 shows that with increasing $\mathrm{RH}$ from $35 \%$ to $80 \%$ the wear factor has first decreased, touched minima, and then increased. The effect of passivation of surface dangling bond by water molecules in ambient conditions is the primary reason behind this phenomenon. The water vapor in the air passivated [26] the dangling bonds on the contact surface of the DLN film through adsorption and/or dissociation mechanisms [26]. This passivation reduced the adhesion interaction with the DLN film and ball surface. This mechanism caused by the presence of water vapor was applicable not only for the DLN coating surface but also for the transferred layer on the contact surface of the ball. When the percentage of water vapor was low (in 35\% RH) in the test chamber, a strong adhesion interaction grew between counter faces, since the passivation of dangling bonds on DLN surface by water vapour was less. This caused higher value of wear factor at less humid air. The COF remained low, as the surface of the DLN film became smoother, and the debris size was in the order of 2-4 micrometer as seen by high resolution optical microscope (Figure 11).

It was observed that when the ball-on-disc test was started in ambient air with 50\% $\mathrm{RH}$, maximum number of dangling bonds got passivated via adsorption of water molecules, and interaction between counter faces was very low which led to low wear factor of DLN film. Adsorption of water molecule on DLN surface also led to dissociation and oxidation of DLN surface $[15,26,27]$. These were transferred to the ball as charged or uncharged fragments. The charged fragments released charges on ball and fell on the wear track as debris. Moreover the debris formed due to sliding agglomerated became larger as RH increased as seen by high resolution optical microscope (Figure 11). The average debris size at $60 \%$ RH condition was $15-17 \mu \mathrm{m}$, whereas it was $2-4 \mu \mathrm{m}$ at $35 \%$ $\mathrm{RH}$. Debris in the sliding interface deformed during sliding. Greater amount of energy was required for deforming larger debris than that for small scattered ones [26, 27]. This caused increase in COF, with increasing $\mathrm{RH}$.

With a further increase in $\mathrm{RH}$ up to $80 \%$ the adhesion interaction was nullified, as dangling bonds were saturated by adsorbing the water molecule from ambient. As the ambient condition encompassed a large percentage of water, hence physisorption of water molecules led to oxidation of DLN surface [28]. Due to this oxidation of the outer most surface, the bonding strength with immediately beneath layer becomes weaker. Hence, the wearing phenomenon occurred more easily during sliding of WC ball. Moreover, 


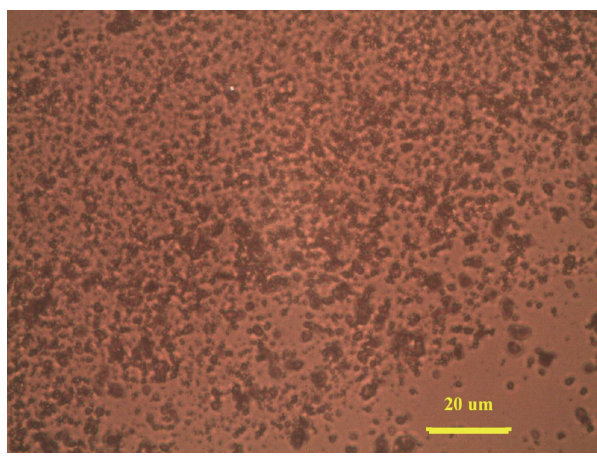

(a)

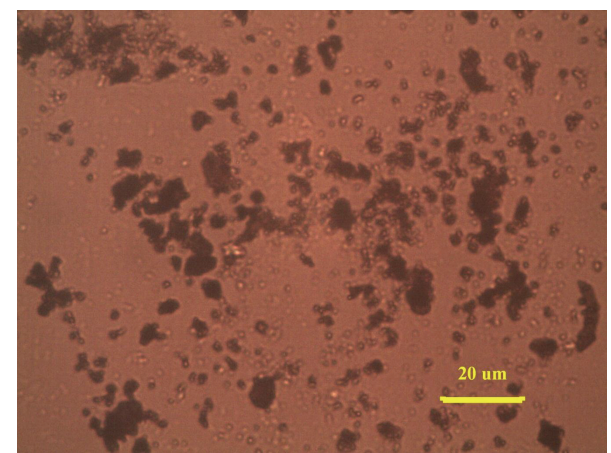

(b)

FIGURE 11: High resolution optical microscope (100X) image of debris produced by wearing in presence of air with (a) $35 \%$ RH and (b) $60 \%$ $\mathrm{RH}$.

TABLE 2: wear rate, wear factor, and COF of DLN film of thickness $1.09 \mu \mathrm{m}$ in different ambient conditions.

\begin{tabular}{lccc}
\hline Ambient & Cycles/micron & Wear factor $\left(\mathrm{mm}^{3} / \mathrm{Nm}\right)$ & Average COF during film life time \\
\hline $35 \%$ RH air & $48200 \pm 2453$ & $9.4 \times 10^{-8} \pm 4.78 E-09$ & $0.005 \pm 0.001$ \\
$40 \%$ RH air & $65500 \pm 5423$ & $6.9 \times 10^{-8} \pm 5.71 E-09$ & $0.011 \pm 0.002$ \\
$50 \%$ RH air & $168687 \pm 7534$ & $2.7 \times 10^{-8} \pm 1.21 E-09$ & $0.021 \pm 0.002$ \\
$60 \%$ RH air & $73833 \pm 5201$ & $6.1 \times 10^{-8} \pm 4.30 E-09$ & $0.055 \pm 0.005$ \\
$80 \%$ RH air & $72000 \pm 4865$ & $6.2 \times 10^{-8} \pm 4.19 E-09$ & $0.074 \pm 0.005$ \\
DI Water & $142100 \pm 11349$ & $3.2 \times 10^{-8} \pm 2.56 E-09$ & $0.057 \pm 0.004$ \\
Saline Soln. & $245500 \pm 12734$ & $1.8 \times 10^{-8} \pm 9.34 E-10$ & $0.021 \pm 0.004$ \\
\hline
\end{tabular}

with increase in $\mathrm{RH}$ the debris size also increased, which in turn enhanced abrasive wear of the film as well as the COF.

In DI water ambient, the COF was less than that for air with high humidity, RH $80 \%$, and closely matched with $60 \%$ $\mathrm{RH}$ air. The wear out debris contained broken pieces of DLN surface peaks, and the tribological byproducts (fragments) all floated in water. Water, in this particular case also acted as a lubricant and coolant. Thus, the COF lowered in DI water as compared to $80 \% \mathrm{RH}$ air. It may be noted that both the COF and wear factor in DI water matched with moderate humidity ambient (RH 50\%-60\%).

In dilute solution of $\mathrm{NaCl}(0.9 \%)$, the $\mathrm{COF}$ was found to have very low value in comparison to $50 \% \mathrm{RH}$ air, and the wear factor was also very low, even lower than that for $50 \%$ $\mathrm{RH}$ air. The reason behind this was, like DI water, saline water, also worked as a lubricant and coolant to reduce friction. In this case also, the debris floated leading to further reduction of friction. Moreover, the saline solution had a much higher electrical conductivity than DI water. The opposite charges that were built up at the two sliding surfaces due to friction were easily discharged via the saline solution. Hence, the adhesive force due to electrical effect reduced drastically resulting in very low wear factor, even less than that for $50 \%$ $\mathrm{RH}$ air.

\section{Conclusion}

DLN films deposited in a PACVD system from a suitable organosilicon precursor have excellent frictional and wear properties. In low humidity condition, the frictional coefficient is extremely low, and the wear factor was relatively high $\left(9.4 \times 10^{-8} \mathrm{~mm}^{3} / \mathrm{Nm}\right)$. The wear factor, however, dipped to a very low value $\left(2.7 \times 10^{-8} \mathrm{~mm}^{3} / \mathrm{Nm}\right)$ at $50 \% \mathrm{RH}$. But the $\mathrm{COF}$ rose to 0.02 . The wear factor was increased by a factor of 3.5 in dry air in reference to $50 \% \mathrm{RH}$ condition. DI water and saline water $(0.9 \% \mathrm{NaCl}$ solution) acted as lubricant and coolant, to reduce wear rate. But friction was higher in DI water than in $\mathrm{NaCl}$ solution. The tribological properties of DLN film were extremely congenial in the ambience of dilute $\mathrm{NaCl}$ solution (0.9\%). This type of solution is isotonic with the contents of human red blood cells. Thus DLN coating may be highly suitable for use in components for artificial hip joint and knee joint replacements. It may also be suitable as solid lubricant for machine parts.

\section{Acknowledgments}

The authors gratefully acknowledge the DST, Government of India for financial support. The XRD, FESEM, and AFM studies were carried out in the IIT, Kharagpur, IACS, Kolkata, and University of Calcutta, Kolkata, respectively.

\section{References}

[1] S. Meskinis and A. Tamuleviciene, "Structure, Properties and Applications of Diamond like Nanocomposite $\left(\mathrm{SiO}_{x}\right.$ Containing DLC) Films: A Review," Materials Science, vol. 17, pp. 358$370,2011$.

[2] C. Venkatraman, A. Goel, R. Lei, D. Kester, and C. Outten, "Electrical properties of diamond-like nanocomposite coatings," Thin Solid Films, vol. 308-309, no. 1-4, pp. 173-177, 1997. 
[3] W. J. Yang, Y. H. Choa, T. Sekino, K. B. Shim, K. Niihara, and K. H. Auh, "Structural characteristics of diamond-like nanocomposite films grown by PECVD," Materials Letters, vol. 57, no. 21, pp. 3305-3310, 2003.

[4] S. Jana, S. Das, U. Gangopadhyay, P. Ghosh, and A. Mondal, "Frequency response of diamond like nanocomposite thin film based MIM capacitor and equivalent circuit modelling," IOSR JEEE, vol. 1, pp. 46-50, 2012.

[5] V. F. Dorfman, A. Bozhko, B. N. Pypkin et al., "Diamond-like nanocomposites: electronic transport mechanisms and some applications," Thin Solid Films, vol. 212, no. 1-2, pp. 274-281, 1992.

[6] K. J. Schoen, J. M. Woodall, A. Goel, and C. Venkatraman, "Electrical properties of metal-diamond-like-nanocomposite (Me-DLN) contacts to 6H SiC," Journal of Electronic Materials, vol. 26, no. 3, pp. 193-197, 1997.

[7] D. Neerinck, P. Persoone, M. Sercu, A. Goel, D. Kester, and D. Bray, "Diamond-like nanocomposite coatings (a-C:H/a-Si:O) for tribological applications," Diamond and Related Materials, vol. 7, no. 2-5, pp. 468-471, 1998.

[8] A. Pandit and N. P. Padture, "Interfacial toughness of diamondlike nanocomposite (DLN) thin films on silicon nitride substrates," Journal of Materials Science Letters, vol. 22, no. 18, pp. 1261-1262, 2003.

[9] W. J. Yang, K. H. Auh, C. Li, and K. Niihara, "Microstructure characteristics of diamond-like nanocomposite (DLN) film by thermally activated chemical vapor deposition," Journal of Materials Science Letters, vol. 19, no. 18, pp. 1649-1651, 2000.

[10] T. Das, D. Ghosh, T. K. Bhattacharyya, and T. K. Maiti, "Biocompatibility of diamond-like nanocomposite thin films," Journal of Materials Science, vol. 18, no. 3, pp. 493-500, 2007.

[11] A. Nath, A. Das, L. Rangan, and A. Khare, "Bacterial inhibition by $\mathrm{Cu} / \mathrm{Cu}_{2} \mathrm{O}$ nanocomposites prepared via laser ablation in liquids," Science of Advanced Materials, vol. 4, no. 1, pp. 106-109, 2012.

[12] S. J. Park, K.-R. Lee, and D.-H. Ko, "Tribochemical reaction of hydrogenated diamond-like carbon films: a clue to understand the environmental dependence," Tribology International, vol. 37, no. 11-12, pp. 913-921, 2004.

[13] S. I. U. Ahmed, G. Bregliozzi, and H. Haefke, "Microfrictional properties of diamond-like carbon films sliding against silicon, sapphire and steel," Wear, vol. 254, no. 11, pp. 1076-1083, 2003.

[14] D. Neerinck, P. Persoone, M. Sercu et al., "Diamond-like nanocomposite coatings for low-wear and low-friction applications in humid environments," Thin Solid Films, vol. 317, no. 1-2, pp. 402-404, 1998.

[15] T. W. Scharf, J. A. Ohlhausen, D. R. Tallant, and S. V. Prasad, "Mechanisms of friction in diamondlike nanocomposite coatings," Journal of Applied Physics, vol. 101, no. 6, Article ID 063521, 2007.

[16] X. Z. Ding, F. M. Zhang, X. H. Liu et al., "Ion beam assisted deposition of diamond-like nanocomposite films in an acetylene atmosphere," Thin Solid Films, vol. 346, no. 1, pp. 82-85, 1999.

[17] M. Park, C. W. Teng, V. Sakhrani et al., "Optical characterization of wide band gap amorphous semiconductors ( $a$-Si:C:H): effect of hydrogen dilution," Journal of Applied Physics, vol. 89, no. 2, pp. 1130-1137, 2001.

[18] R. E. Shroder, R. J. Nemanich, and J. T. Glass, "Analysis of the composite structures in diamond thin films by Raman spectroscopy," Physical Review B, vol. 41, no. 6, pp. 3738-3745, 1990.
[19] F. Tuinstra and J.L. Koenig, "Raman spectroscopy of graphite," Journal of Chemical Physics, vol. 53, no. 3, pp. 1126-1130, 1970.

[20] R. O. Dillon, J. A. Woollam, and V. Katkanant, "Use of Raman scattering to investigate disorder and crystallite formation in asdeposited and annealed carbon films," Physical Review B, vol. 29, no. 6, pp. 3482-3489, 1984.

[21] C. Casiraghi, F. Piazza, A. C. Ferrari, D. Grambole, and J. Robertson, "Bonding in hydrogenated diamond-like carbon by Raman spectroscopy," Diamond and Related Materials, vol. 14, no. 3-7, pp. 1098-1102, 2005.

[22] K. Honglertkongsakul, P. W. May, and B. Paosawatyanyong, "Electrical and optical properties of diamond-like carbon films deposited by pulsed laser ablation," Diamond and Related Materials, vol. 19, no. 7-9, pp. 999-1002, 2010.

[23] J. Z. Wan, F. H. Pollak, and B. F. Dorfman, "Micro-Raman study of diamondlike atomic-scale composite films modified by continuous wave laser annealing," Journal of Applied Physics, vol. 81, no. 9, pp. 6407-6414, 1997.

[24] A. C. Ferrari and J. Robertson, "Interpretation of Raman spectra of disordered and amorphous carbon," Physical Review B, vol. 61, no. 20, pp. 14095-14107, 2000.

[25] S. J. Park, K.-R. Lee, and D.-H. Ko, “Tribological behavior of nano-undulated surface of diamond-like carbon films," Diamond and Related Materials, vol. 14, no. 8, pp. 1291-1296, 2005.

[26] E. Konca, Y.-T. Cheng, A. M. Weiner, J. M. Dasch, and A. T. Alpas, "Vacuum tribological behavior of the non-hydrogenated diamond-like carbon coatings against aluminum: effect of running-in in ambient air," Wear, vol. 259, no. 1-6, pp. 795-799, 2005.

[27] K. Y. Eun, K. R. Lee, E. S. Yoon, and H. Kong, "Effect of polymeric debris on the tribological behavior of diamond-like carbon films," Surface and Coatings Technology, vol. 86-87, no. 2, pp. 569-574, 1996.

[28] S. H. Yang, H. Kong, K. R. Lee, S. Park, and D. E. Kim, "Effect of environment on the tribological behavior of Si-incorporated diamond-like carbon films," Wear, vol. 252, no. 1-2, pp. 70-79, 2002. 

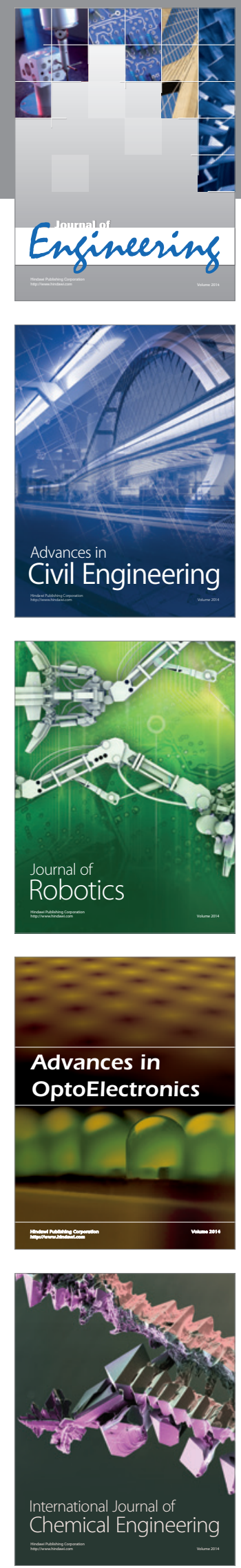

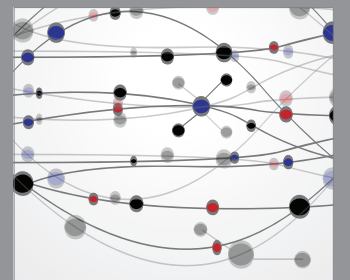

The Scientific World Journal
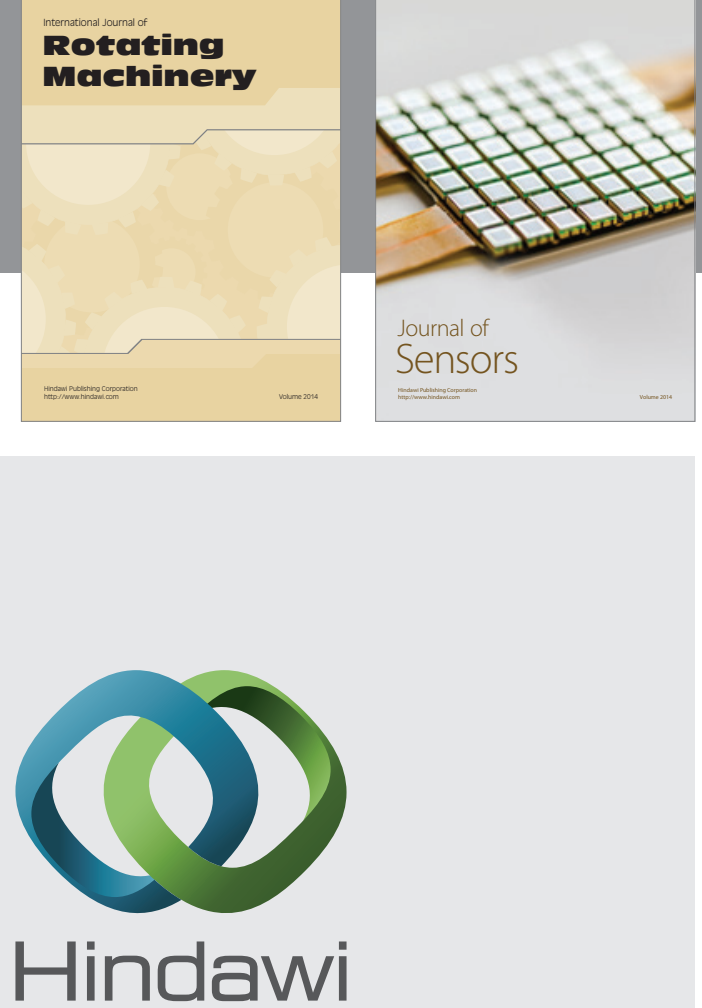

Submit your manuscripts at http://www.hindawi.com
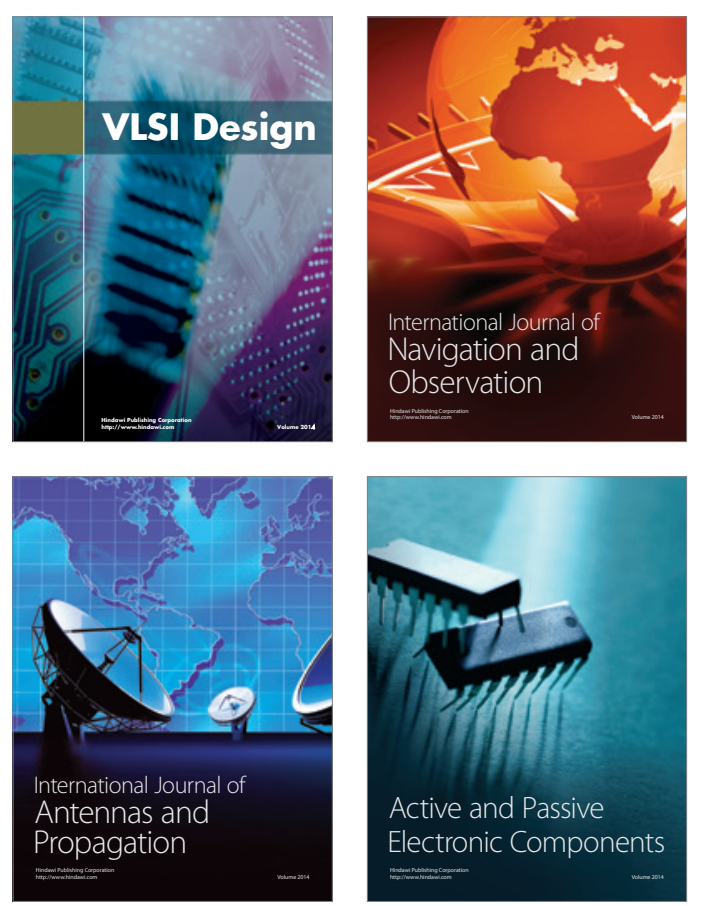
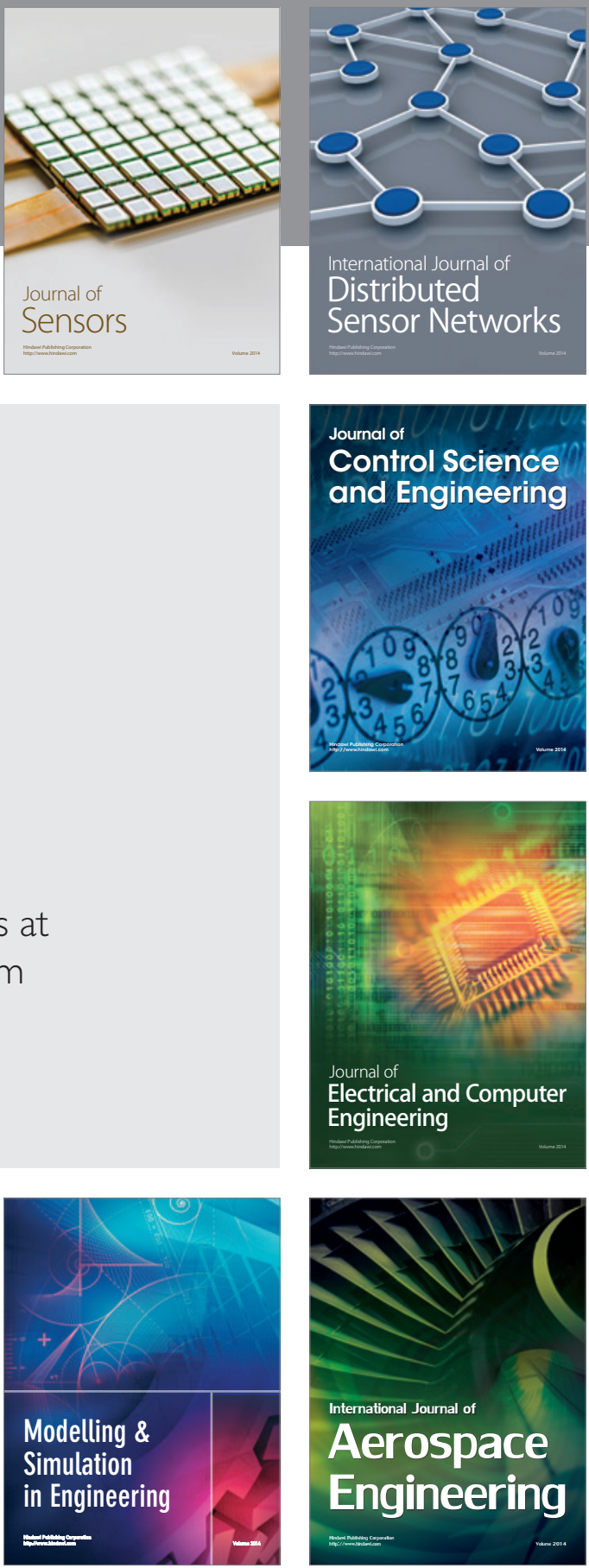

Journal of

Control Science

and Engineering
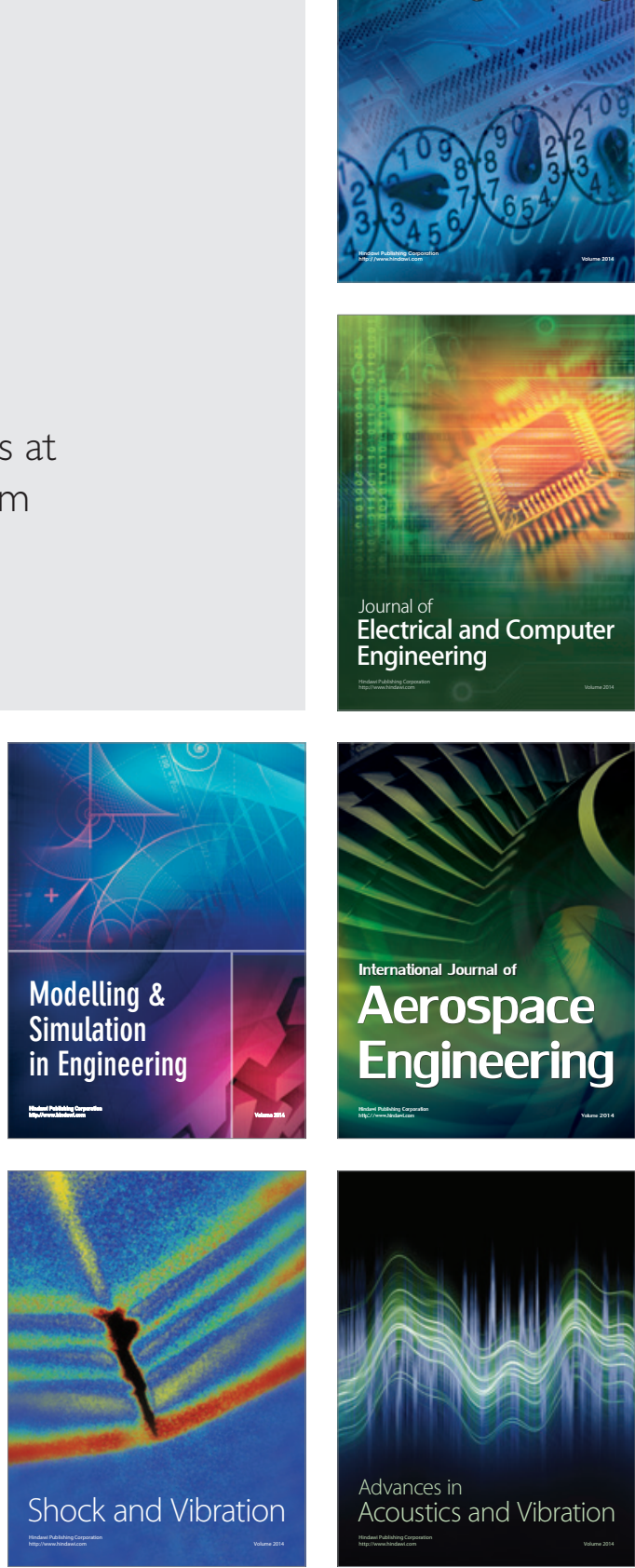